\title{
Quantitative Experimental Study of Intrachannel Nonlinear Timing Jitter in a 10-Gb/s Terrestrial WDM Return-To-Zero System
}

\author{
Hai Xu, John Zweck, Li Yan, Member, IEEE, Curtis R. Menyuk, Fellow, IEEE, and \\ Gary M. Carter, Senior Member, IEEE
}

\begin{abstract}
Using a recirculating loop, we measured nonlinearly induced timing jitter in a terrestrial wavelength-division-multiplexed system at different transmission distances with different amounts of precompensation. Within each 600-GHz subband, we achieved error-free transmission using the same amount of precompensation for all channels at all distances up to $5000 \mathrm{~km}$.
\end{abstract}

Index Terms-Nonlinear distortion, optical fiber communication, optical fiber dispersion, optical Kerr effect, timing jitter.

\section{INTRODUCTION}

$\mathbf{I}^{N}$ $\mathrm{N}$ return-to-zero (RZ) optical communication systems, timing jitter induced by intrachannel nonlinear pulse-to-pulse interactions can cause significant performance degradation [1]. Studies have shown that the amount of timing jitter depends on the predispersion and postdispersion compensation and the dispersion map [2]-[8]. However, no quantitative experimental characterization of the timing jitter in a long-haul terrestrial wavelength-division-multiplexed (WDM) system and its impact on the system performance has been reported.

In this letter, we report on an experimental study of timing jitter as a function of transmission distance up to $5000 \mathrm{~km}$ with different amounts of precompensation. We use a recirculating loop to emulate a 10-Gb/s (WDM) terrestrial system. We quantify the dependence of the timing jitter on the transmission distance and the precompensation, and we determine its impact on the bit-error rate (BER). We show that by choosing a fixed amount of precompensation within each $600-\mathrm{GHz}$-wide subband of the $C$-band, we can limit the timing-jitter-induced BER degradation and achieve error-free propagation (BER $<1 \times$ $10^{-9}$ ) for all distances up to $5000 \mathrm{~km}$.

Previous studies of single-span systems with lossless fiber show that to minimize timing jitter due to intrachannel nonlinear interactions, the precompensation and postcompensation should be symmetric, i.e., the amounts of predispersion and postdispersion compensation should be equal [2], [3]. However, this simple symmetry rule does not hold for a single-span system with loss [3]-[5]. On the other hand, a long-haul transmission system might have different characteristics. For example, theoretical studies of long-haul point-to-point undersea systems, in

Manuscript received July 1, 2003; revised August 22, 2003. This work was supported by the CIENA Company.

The authors are with the Computer Science and Electrical Engineering Department, University of Maryland Baltimore County, Baltimore, MD 21250

USA (e-mail: hxu@engr.umbc.edu).

Digital Object Identifier 10.1109/LPT.2003.820470

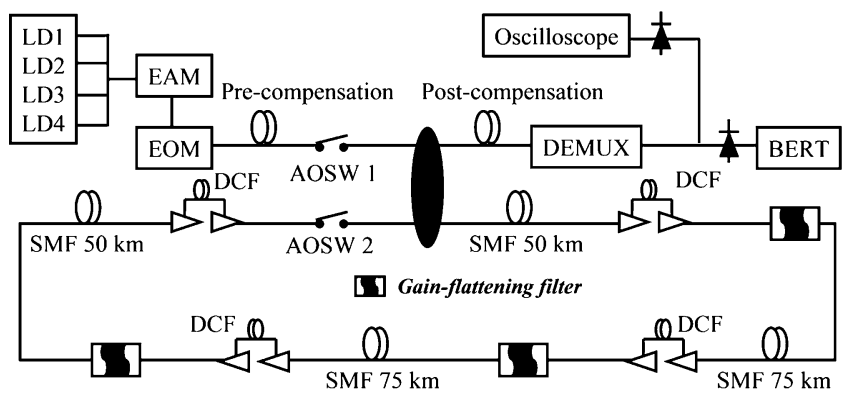

Fig. 1. Experimental setup.

which the accumulated dispersion in each span of fiber is small compared to the accumulated dispersion in the whole transmission line, show that symmetric precompensation and postcompensation minimizes the timing jitter even when fibers have nonzero loss [6], [7]. Furthermore, in many terrestrial transmission systems, the accumulated dispersion in each span of transmission fiber can be comparable to the accumulated dispersion in the whole transmission line. We show that in this case, the optimal precompensation and postcompensation satisfy a modified symmetry rule.

\section{EXPERIMENTAL SETUP}

Fig. 1 shows our recirculating loop that consisted of four transmission spans of standard single-mode fiber [(SMF) $D=$ $+17.4 \mathrm{ps} / \mathrm{nm} \cdot \mathrm{km}]$. Following each span of SMF, there was a compensation module that included a dual-stage erbium-doped fiber amplifier (EDFA) and partially slope-compensating dispersion compensating fiber [(DCF) $D=-97 \mathrm{ps} / \mathrm{nm} \cdot \mathrm{km}$ ]. The length of SMF was about $50 \mathrm{~km}$ in the first and fourth spans and about $75 \mathrm{~km}$ in the second and third spans. The total length of SMF in the loop was $260 \mathrm{~km}$. We transmitted four equally spaced channels between 1552.6 and $1557.4 \mathrm{~nm}$. Gainflattening filters were used in the loop to compensate for the uneven gain profile of the EDFAs. We passed the four channels through an electroabsorption modulator to produce $10-\mathrm{Gb} / \mathrm{s}$ RZ pulses with a full-width at half-maximum of $21 \mathrm{ps}$ and then modulated the pulses with a pseudorandom data of length $2^{23}-$ 1 . The average power per channel was about $-2.5 \mathrm{dBm}$ into the SMF and $-6.5 \mathrm{dBm}$ into the DCF. We used a length of SMF or DCF for the predispersion and postdispersion compensation. By varying the average loop dispersion with a short piece of SMF or DCF, we used the four fixed-wavelength channels to 


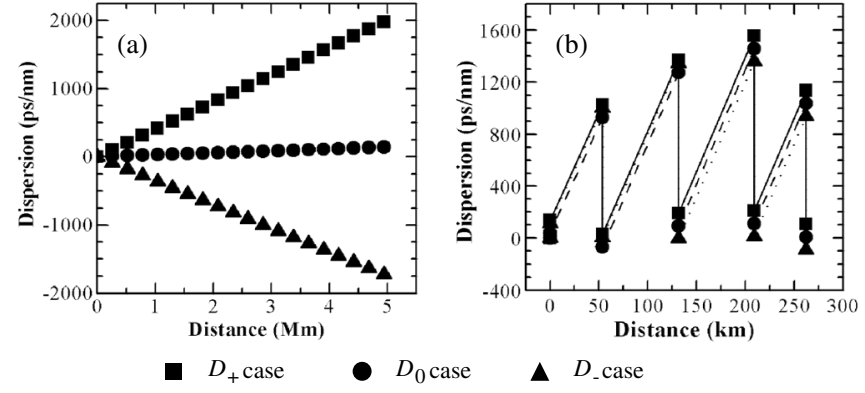

Fig. 2. (a) Accumulated transmission dispersion for the channel at $1557.4 \mathrm{~nm}$, measured after each roundtrip excluding precompensation and postcompensation. (b) Accumulated dispersion in one roundtrip.

study different subbands in the $C$-band, which, for a fixed dispersion map, is equivalent to varying the wavelength. In this experiment, we focused on three cases for which the average loop dispersion of channel 4 (1557.4 nm) was $-0.29,0.02$, and $+0.33 \mathrm{ps} / \mathrm{nm} \cdot \mathrm{km}$ respectively. These three cases, which we call the $D_{-}, D_{0}$, and $D_{+}$cases, correspond to the short, central, and long wavelength regions of the $C$-band in which the central wavelength channel experiences zero average transmission dispersion. The average dispersion slope of the loop was around $+0.017 \mathrm{ps} / \mathrm{nm}^{2} \cdot \mathrm{km}$. In Fig. 2, we show the accumulated transmission dispersion, and the local dispersion maps for these three cases. We refer to the dispersion accumulated in a single span of SMF as the accumulated span dispersion $D_{\text {span }}$, and we refer to the accumulated dispersion measured after each roundtrip, excluding the precompensation and postcompensation, as the accumulated transmission dispersion $D_{\text {trans. }}$.

We used an Agilent sampling oscilloscope to record the standard deviation of the timing shift of the signal before transmission $\left(\sigma_{0}\right)$ and after transmission $\left(\sigma_{T}\right)$ and calculated the timing jitter induced in transmission to be $\sqrt{\sigma_{T}^{2}-\sigma_{0}^{2}}$. We used an Anritsu BER tester in burst mode to measure the BER.

\section{Results AND Discussions}

Fig. 3 shows the measured timing jitter of channel 4 $(1557.4 \mathrm{~nm})$ at different transmission distances for each dispersion case. The other three channels have similar characteristics. For each amount of precompensation and at each distance at which we measured the timing jitter, we adjusted the amount of postcompensation to minimize the BER. With the optimal precompensation and with postcompensation adjusted to minimize the BER, the total amount of dispersion was between -100 and $+100 \mathrm{ps} / \mathrm{nm}$. The timing jitter is mostly caused by intrachannel nonlinear effects. We verified that interchannel effects are negligible by checking that the system performance changed only a little when we increased the channel spacing beyond $200 \mathrm{GHz}$. In Fig. 3, we see that without proper precompensation, the timing jitter can be as large as 9.3 ps at $3500 \mathrm{~km}$, and this amount of timing jitter prevents error-free propagation. Furthermore, the timing jitter evolution is different in the three cases. However, in all three cases, with the optimal amount of precompensation, we can minimize the timing jitter and propagate the signal error-free for all distances up to $5000 \mathrm{~km}$. In Fig. 4, we show the measured optimal amount of precompensation of channel 4 for all three cases.
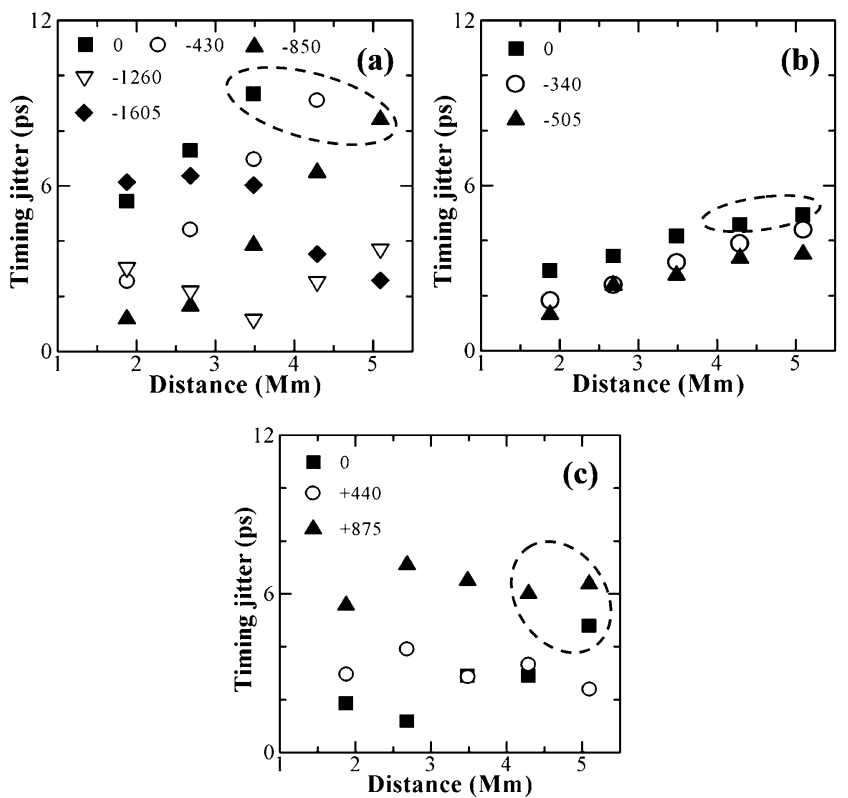

Fig. 3. Timing jitter versus transmission distance for various amounts of precompensation for the channel at $1557.4 \mathrm{~nm}$ (shown in insets with units of picoseconds per nanometers). (a) Case $D_{+}$. (b) Case $D_{0}$. (c) Case $D_{-}$. For the points circled with dashes, the system was not error free no matter how we adjusted the postcompensation.

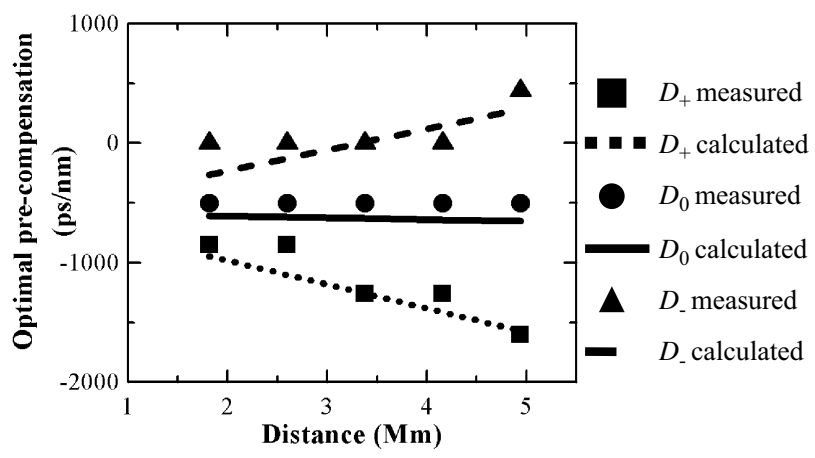

Fig. 4. Comparison of the measured and calculated optimal amounts of precompensation for the channel at $1557.4 \mathrm{~nm}$.

For the $D_{+}$and $D_{-}$cases, during transmission, the pulses are sufficiently broad so that the amplitude jitter due to selfphase modulation is small. On the other hand, unlike in some $40-\mathrm{Gb} / \mathrm{s}$ systems, the pulses within a channel do not overlap strongly enough during transmission to result in amplitude jitter due to intrachannel four-wave mixing [1], [8]. In the $D_{0}$ case, without precompensation, the amplitude jitter induced by selfphase modulation limits the system performance because the pulses have a narrow width and a large peak power where the average power is largest. In the $D_{0}$ case, this amplitude jitter is greatly reduced at the optimal precompensation.

As pointed out in [1] and [2], the physical mechanism by which timing jitter is minimized at a fixed distance is that, with a proper amount of precompensation, the average accumulated dispersion changes sign about half way from transmitter to receiver. This ensures that the contribution to the timing jitter produced in the first half of the transmission is almost cancelled in the second half of the transmission. In Fig. 3(a) and (c), we observe this timing jitter cancellation in the $D_{+}$and $D_{-}$cases 


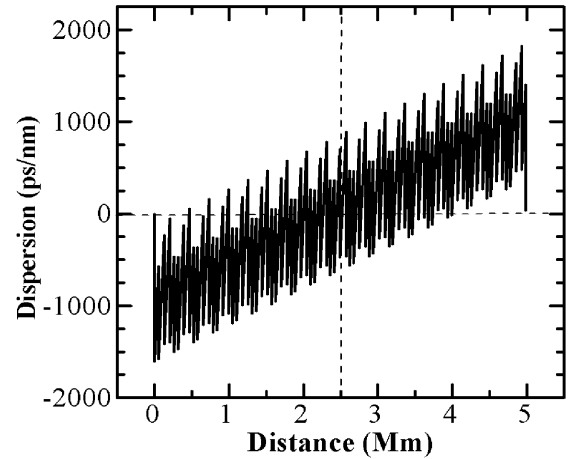

Fig. 5. Accumulated dispersion that minimizes the timing jitter for the channel at $1557.4 \mathrm{~nm}$ at $5000 \mathrm{~km}$ in the $D_{+}$case with $-1604 \mathrm{ps} / \mathrm{nm}$ of precompensation. Both precompensation and postcompensation are included.

when the precompensation is chosen to minimize the timing jitter at longer distances.

In many terrestrial systems, the accumulated span dispersion $D_{\text {span }}$ is comparable to the accumulated transmission dispersion $D_{\text {trans }}$. Consequently, the optimal amount of precompensation does not follow the simple symmetry rule discussed above [6], [7]. As shown in Fig. 2, in the $D_{+}$and $D_{-}$cases, at a transmission distance of $5000 \mathrm{~km}$, the accumulated transmission dispersion $D_{\text {trans }}$ is +1980 and $-1730 \mathrm{ps} / \mathrm{nm}$, but the optimal amount of precompensation is -1605 and $+440 \mathrm{ps} / \mathrm{nm}$, respectively.

In the terrestrial system we studied, the local dispersion map is not symmetric, and the timing jitter induced in one map is larger than that in a symmetric map. By a symmetric dispersion map, we mean one for which the dispersion accumulated within the map is close to zero at the midpoint of the map as well as at the beginning and the end of the map. The optimal amount of precompensation, thus, consists of two parts: One part is used to minimize the timing jitter induced in one map and the amount is $-D_{\text {span }} / 2$; the other part minimizes the timing jitter associated with the accumulated transmission dispersion and the amount is $-D_{\text {trans }} / 2$. Thus, the total optimal amount of precompensation required to minimize the timing jitter $D_{\text {pre,opt }}$ can be approximated by

$$
D_{\text {pre }, \text { opt }}=\frac{-\left(\overline{D_{\text {span }}}+D_{\text {trans }}\right)}{2}
$$

where $\overline{D_{\text {span }}}$ is the average accumulated span dispersion, which for our system is $\overline{D_{\text {span }}} \approx+1164 \mathrm{ps} / \mathrm{nm}$. The calculated optimal amounts of precompensation, as shown in Fig. 4, agree reasonably well with the measured values in all three cases. This formula for the optimal precompensation results in an accumulated dispersion map that satisfies the following modified symmetry rule: The optimal precompensation is such that the accumulated dispersion function is symmetric with respect to a $180^{\circ}$ rotation about the midway point of the transmission distance. In Fig. 5, we show as an example the accumulated dispersion function in the $D_{+}$case with $-1605 \mathrm{ps} / \mathrm{nm}$ of precompensation, which minimizes the timing jitter at $5000 \mathrm{~km}$. This modified symmetry rule also applies to the systems studied in [6] and [7].
From Fig. 3, we observe that the system is more vulnerable to timing jitter impairments at longer distances than at shorter distances. At $2500 \mathrm{~km}$, the system is error free even when the timing jitter is as large as $7.3 \mathrm{ps}$, as in the $D_{+}$case without precompensation shown in Fig. 3(a). In contrast, at $5000 \mathrm{~km}$, error-free propagation is not possible with a timing jitter of $4.8 \mathrm{ps}$, in the $D_{-}$case without precompensation, as shown in Fig. 3(c). This is because at shorter distances, the amplified spontaneous emission noise is smaller and the system can tolerate more nonlinearly induced degradation. For example, the optical signal-to-noise ratio is around $15.0 \mathrm{~dB}$ at $2500 \mathrm{~km}$ and is about $12.0 \mathrm{~dB}$ at $5000 \mathrm{~km}$. In the $D_{0}$ case, the system performance is less tolerant to timing jitter as there is more amplitude jitter due to self-phase modulation.

With a fixed precompensation of -1260 or $-1605 \mathrm{ps} / \mathrm{nm}$ in the $D_{+}$case, -340 or $-505 \mathrm{ps} / \mathrm{nm}$ in the $D_{0}$ case, and $+430 \mathrm{ps} / \mathrm{nm}$ in the $D_{-}$case, we achieved error-free propagation at all distances up to $5000 \mathrm{~km}$ across the $600-\mathrm{GHz}$-wide subband.

\section{CONCLUSION}

We experimentally characterized the evolution of timing jitter with different amounts of precompensation for channels in different wavelength regions in a $10-\mathrm{Gb} / \mathrm{s}$ long-haul terrestrial RZ WDM system. We found that the optimal precompensation follows a modified symmetry rule that takes into account both the local dispersion map and the accumulated transmission dispersion. We showed that with an appropriate fixed amount of precompensation for each $600-\mathrm{GHz}$-wide subband, error-free transmission can be achieved for all distances up to $5000 \mathrm{~km}$.

\section{ACKNOWLEDGMENT}

The authors would like to thank M. Taylor for advice.

\section{REFERENCES}

[1] V. Mamyshev and N. A. Mamysheva, "Pulse-overlapped dispersion-managed data transmission and intrachannel four-wave mixing," Opt. Lett., vol. 24, pp. 1454-1456, 1999.

[2] A. Mecozzi, C. B. Clausen, M. Shtaif, S. G. Park, and A. H. Gnauck, "Cancellation of timing and amplitude jitter in symmetric links using highly dispersed pulses," IEEE Photon. Technol. Lett., vol. 13, pp. 445-447, May 2001.

[3] J. Martensson, M. Westund, and A. Berntson, "Intra-channel pulse interactions in 40-Gbit/s dispersion-managed RZ transmission system," Electron. Lett., vol. 36, pp. 244-246, 2001.

[4] R.-J. Essiambre, B. Mikkelsen, and G. Raybon, "Intra-channel crossphase modulation and four-wave mixing in high-speed TDM systems," Electron. Lett., vol. 35, pp. 1576-1578, 1999.

[5] R. I. Killey, H. J. Thiele, V. Mikhailov, and P. Bayvel, "Reduction of intrachannel nonlinear distortion in 40-Gb/s-based WDM transmission over standard fiber," IEEE Photon. Technol. Lett., vol. 12, pp. 1624-1626, Dec. 2000.

[6] E. A. Golovchenko, A. N. Pilipetskii, and N. S. Bergano, "Transmission properties of chirped return-to-zero pulses and nonlinear intersymbol interference in $10 \mathrm{~Gb} / \mathrm{s}$ WDM transmission," presented at the OFC/IOOC Conf. 2000, Baltimore, MD, Mar. 2000, Paper FC3.

[7] R.-M. Mu, T. Yu, V. S. Grigoryan, and C. R. Menyuk, "Convergence of the CRZ and DMS formats in WDM systems using dispersion management," presented at the OFC/IOOC 2000, Baltimore, MD, Mar. 7-10, 2000, Paper FC1.

[8] S. Kumar, J. C. Mauro, S. Raghavan, and D. Q. Chowdhury, "Intrachannel nonlinear penalties in dispersion-managed transmission systems," IEEE J. Select. Topics Quantum Electron., vol. 8, pp. 626-631, May/June 2002. 